$\xi=-1$

\title{
Performance evaluation of power optimization in wireless sensor networks using particle swarm optimization
}

\author{
Chandaluru Mohan Venkata Siva Prasad ${ }^{1}$ *, Dr. K. RaghavaRao ${ }^{2}$, D. Satish Kumar ${ }^{3}$, A. V. Prabhu ${ }^{4}$ \\ ${ }^{1}$ M. tech Student, Department of Electronics and Computer Science Engineering \\ ${ }^{2}$ Professor \& Head of the Department, Electronics and Computer Science Engineering \\ ${ }^{3}$ Assistant Professor, Department of Mathematics \\ ${ }^{4}$ Associate Professor, Department of Electronics and Computer Science Engineering Koneru Lakshmiah \\ Education Foundation(Deemed to be University), Guntur, Andhra Pradesh, India, 522502 \\ *Corresponding author E-mail: mohanchandaluru@gmail.co
}

\begin{abstract}
Wireless sensor networks are the sensors which are acclimated to sense the environmental condition like temperature, pressure, sultriness, moisture etc, sensing the environment parameters and sending them to the gateway and retrieving the aggregated data from the gateway to the end user. Power is the major constraint in wireless sensor networks. One must need to reduce the power consumption. Wireless sensor networks have sensor nodes in which each node has a processor, antenna and a battery. The batteries consume power so that we require increasing the lifetime of the battery for that some optimization techniques are required to reduce the power consumption. There are some techniques which are inspired from the lifestyle of animals. In this paper proposing an optimization technique which is inspired by the animal demeanor which reduces the power consumption of the sensor nodes which is particle swarm optimization (PSO) technique. PSO is inspired by the convivial demeanor of birds or schooling of fish. By utilizing this bio-inspired technique we can reduce the power consumed by the sensor nodes and at the same time lifetime of the batteries present in the sensor nodes are increased.
\end{abstract}

Keywords: Bio-Inspired; Cluster; Power Optimization; PSO; Wireless Sensor Networks.

\section{Introduction}

Wireless sensor networks are acclimated to sense the environment in reactive and proactive manner. Considering the reactive way it senses the environment whenever the event is oc- cured or in a desultory manner. Whereas proactive way senses the environment at each and every interval of time. The sensor node senses the environment in two ways mainly, one is reactive and the other is proactive [1]. Reactive betokens sensing the environment in a customary substructure. Proactive denotes sensing the environment in an arbitrary manner. A sensor network has differ- ent challenges to face in the current trends and the nodes are get- ting deployed in different areas to sense different parameters in the environment. The nodes deployed in undergrounds, and also for weather monitoring [2] sensing applications military applications. Wireless sensor networks senses the environment like temperature, pressure, sultriness and so on.., sensing the environ- ment and sending the data to the gateway is the whole process going on. First the data is sensed by the nodes in the network and data is aggregated. Then the aggregated data is send to the gate- way, whenever the end user requires the data at particular timeInstant then the data is retrieved from the gateway to the cessation end user. The data is send from the starting point to the end user through the sensor nodes. This process requires some amount of energy. The energy consumption is dissipated while transmitting the data from a source to destination. Since the sensor nodes deployed in the environment are non-replaceable batteries so if the sensor node is died we require replacing it with other node. Because battery is not replaceable, we cannot power up the batteries.
So, what we can do is to reduce the power consumption of the sensor nodes and then by this we can increase the lifetime of the batteries. The sensor network has a processor unit, an antenna and a memory to store the sensed data [3].

The deployed WSN have many problems after deploy- ing. WSN consists of different components some of the compo- nents may deplete sooner and some may deplete very late depends up on their life time and quality of the network components. Our main motto is to control the power consumed by the network components during their transmission of the data and one more thing one should keep in mind that the lifetime of the batteries used in the network. The batteries which are in the nodes are non-replaceable so we need to increase the lifetime of the batteries in order to get more time working of the nodes without dead.

The central unit of WSN is a Node, likewise called as bit by UC Berkley. Every sensor hub is required to be fit for detecting, preparing and imparting the handled information to the neighboring hubs to frame a system. Sensor hub is henceforth made out of sensors to detect the physical wonder, simple to computerized converter, microcontroller for controlling and information handling, memory for calculations and information stockpiling, radio unit for short range remote correspondence and battery unit to control every one of the units. For some applications, if possible, sun based fueled chargeable battery units are utilized to limit the disappointment of sensors and support cost.

Data from starting point to the end user. There are so many techniques are there to optimize the potency. We can optimize the power by making all the sensor nodes present in the network into clusters. 
There are some bio-inspired algorithms of optimization techniques depends on the population substructure. Bio-inspired and deportment population predicated optimization techniques to reduce the puissance consumption in the WSN [4]. The different algorithms which are getting inspired by the life style of animals are chosen to optimize the energy efficiency in the nodes of the networks.

The objectives of WSN are:

1) The vigor and unwavering quality of the whole correspondence framework should be enhanced without expanding the vitality utilization.

2) Novel ideas in convention configuration will be exam- ined to additionally stretch out the wake-up framework to likewise impart parameter changes (a couple of byte) to the portable hubs. For accomplishing this objective, the learning on the example of the arriving movement into a system is required.

3) The arrangement of the power sparing components and their right pre setup.

4) Protocol choices between the portable hubs and to en- hance the unwavering quality of the whole correspondence framework while keeping up the officially tight vitality spending plan.

5) To create novel and exact systematic models to break down the execution of the proposed conventions and transmission plans. The exactness of the created models ought to likewise be checked.

One approach can be habituated to control the amount power consumed in WSN is to make the cluster formation of the sensor nodes and each cluster has a head. First the data is sensed by the sensors and then the sensors transmit the data to its mote then mote is the responsible thing to transmit the data packet to the destination. Anteriorly the data is sensed by the sensors and these sensors transmit the data to their neighboring sensors until it reaches the destination. This whole process consumes more power. Later all the sensor nodes composed different groups called Clusters. Each cluster has some sensors and a single head called cluster head. Now the data is sensed by the sensors in the cluster and the sensed data is transmitted to their heads. These heads transmits the data to the base station and from base station to the destination point.

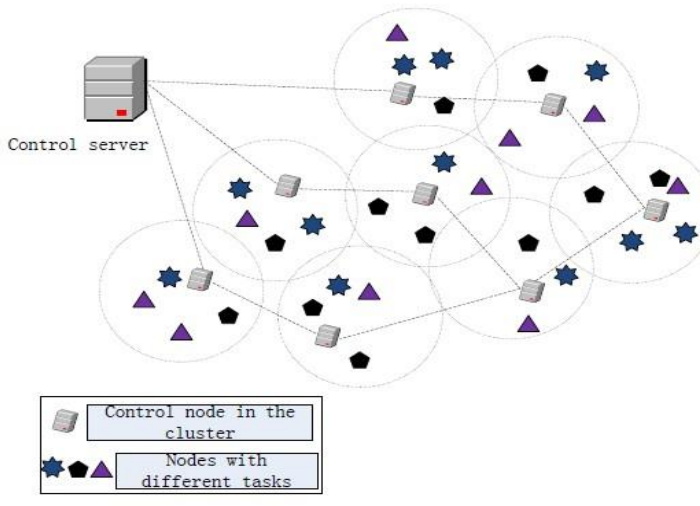

Fig. 1: Wireless Sensor Networks Cluster.

Power optimization is the process of reducing the re- quired amount of power that a sensor node requires to transmit the In recent years researches are going on in different algo- rithms based on the different animal's lifestyle and by that inspired lifestyle we are going to develop an algorithm and by using that particular algorithm we can reduce the power in WSN by applying such techniques.

In this paper we are proposing an optimization technique which is population predicated convivial deportment of animal's algorithm. The particle swarm optimization technique is predicat- ed on the swarm astuteness. Flock of birds or schooling of fish. Each bird in the swarm is considered as a particle or solution quandary and each particle has a fitness value. Velocity which shows the oversight to reach the destination. Position of the particle. Each parti- cle has its personal best value called pbest and all the particles in the swarm has an ecumenical best fitness value called gbest. This technique is better than the existed algorithm based on ant's life style. The ant's life style gives the shortest path for the destination [5]. But this algorithm gives the optimization solution for the problem.

\section{Literature survey}

M.Sasikala [6] proposed some clustering algorithms of wireless sensor networks. In this paper the author embodied that wireless sensor networks are the emerging technology now a days and the data aggregation done through wireless sensor networks is having the quality and the nodes in the network senses and aggre- gating the data requires power. Power is the major constraint in the wireless sensor networks. By the clustering of the sensor nodes one can reduce the power required for the nodes to transmit the data. By multi hop we can reduce the power consumption. The overview of routing protocols is given in this paper.

Mukul pratap singh and Kunal gupta penned the optimi- zation techniques for reduction of power consumption in [7]. The batteries are non-rechargeable and power is the major constraint in wireless sensor networks and for that to reduce the power con- sumption optimization techniques are involved in this paper are like Ants Colony Optimization(ACO) and some other techniques like LEACH, HEED also explained in this paper. ACO is the better technique among those which gives us the shortest path from source to destination.

Chandni and Anjali Bharti explained the bio inspired al- gorithms used to reduce the power consumption in [8] now a days research is going on wireless sensor networks. There are some challenges in wireless sensor networks includes cost, power, and life- time. Power is the major constraint the bio inspired algorithms which reduces the power consumption in networks are brief out in this particular paper.

Salma given a new optimization technique based on grouping of the sensor nodes in [9]. In networks grouping the sensor nodes will reduce the power consumption. The nodes are grouped and form a cluster and each cluster has a head called a mote the group members sense the data and informed to the mote and then the mote sends the data to the neighbor motes and it takes the responsibility of data aggregation and transmission. By this grouping of sensors optimization can be takes place.

The most important issue in the WSN is optimization of minimum resources. Dehghani in [10] proposed many routing algorithms are penned and described in detail for the optimization purpose. Some genetic algorithms also explained in this paper for the power optimization purpose. Power is the major hottest topic for the researchers in wireless sensor networks and it is the major challenge for them in their research area.

Vimala Rani, Subbrahmanian proposed a bio inspired algorithm for the power optimization in WSN called enhancement of particle swarm optimization technique [11]. This technique is bio inspired technique by the flock of birds and school of fishing. The lifestyle of the birds is inspired to follow up this technique. By this technique power can be optimized.

Debmalya Bhattacharya in [12] explained about the issues related to power in the present networks challenges faced by many researchers in WSN. The major constraint is power. For the optimization purpose some algorithms are explained in the paper.

T. Luo, H.-P. Tan and T. Q. Quek in [13] explained about the WSN how they are going to sense the environment and what the different ways to sense the environment are. The challenges that might have been faced in the WSN.

Sandra Sundra in [14] given distinctive calculations in view of the way of life of the creature conduct and by the conduct we can apply those procedures to the systems and after that we can limit the power utilization in the hubs

Debmalya Bhattacharya and R Krishna Moorthy in [15] clarified about the WSN and how the different hubs are conveyed in the 
earth and how they will detect nature they said some unique strategies which limits the cost and builds the lifetime of the hubs.

G.Hemavathy, S.Prabhu in [16] clarified that the WSN comprises of various kinds of various sensors and which are ac- claimed to detect the earth of various physical parameters. These creators clarified that there are two calculations which are utilized to allot the vitality to the hubs by figuring the separation and de- pending up on the separation the hubs are getting assigned some vitality and by that we can diminish the power utilization required for transmission of information parcel.

MM Chandane in [17] clarified different issues that are looking in WSN and the difficulties incorporates into WSN how these difficulties are overcome in WSN he penned a few calcula- tions with the end goal of improvement in WSN hubs.

\section{Proposed approach}

In this paper proposing an optimization technique which is based on bird's life style. This technique is predicated on the swarm intelligence Flock of birds or schooling of fish.[12]. Each bird in the swarm is considered as a particle or solution quandary and each particle has a fitness value. Velocity which shows the oversight of the path to reach the destination. Position of the parti- cle. Each particle has its personal best value called pbest and all the particles in the swarm has an ecumenical best fitness value called gbest. [12]

PSO was introduced and developed by Eberhart and Kennedy in 1995. PSO is multi objective and dynamic optimiza- tion. It is one of the algorithms taken from inspiration of animals in which flock of animals. In flock of animals find aliment desul- torily which is most proximate to victuals position. Animal's up- date to each other about position of food. It will update again and again until food source is found. Particle swarm optimization con- sists of a group of particles, where particle represent a potential

Solution. Every particle is represented by its best position. It calcu- lated on substructure of local best position as lbest and global best position gbest. PSO calculated on position of particle. Position of the particle is getting updated at every repetition.

By the way of life of feathered creatures this calculation is produced and is utilized for the enhancement reason in the sen- sor hubs. The accompanying areas give a concise clarification how the flying creature's way of life is taken as a motivation and how it is connected and contrasted with our systems. What are all the conceivable terms in that calculation are clarified unmistakably in following areas.

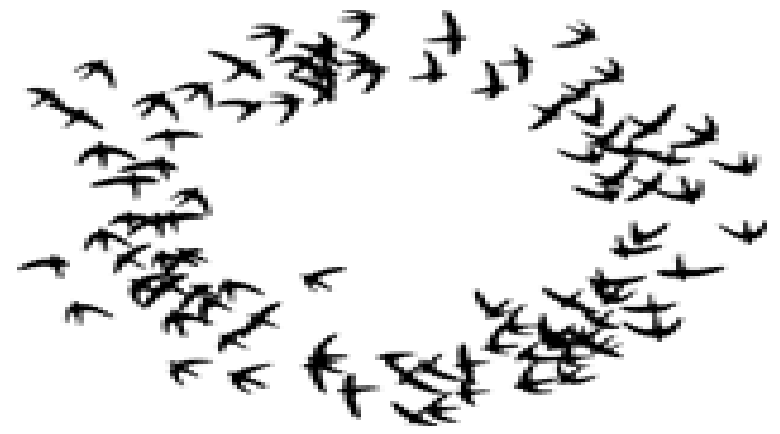

Fig. 2: Bird Flock in the Sky.

We as a whole know how flying creatures are flying in the sky day by day we are watching them while they are move starting with one place then onto the next. There is a similitude between the winged creatures relocating from one point to other point and the hubs which are transmitting the information to one point to other point by this comparability.

Birds move in one angle from source point to their des- tination point for the food particles as appeared in figure 2 every one of the flying creatures in the sky or the gathering doesn't know the area of food however one among all is closer to the food parti- cle. The bird nearer to that bird started following the path until they reach the food particle.

A question is raised to all how we will analyze this feathered creature way of life or the run of winged creatures way of life of looking for the food particles to our system. Yes we can compare. Presently let us contrast the flying creature way of life and the system. Each bird is considered as a sensor in our system and group of birds is called a flock a group of sensors is called as a cluster. Each bird having a position and a velocity vector which shows the path for the birds and here in every sensor additionally we will think about the position of the sensor and having a speed vector for heading of the way to transmit the information. At each cycle the birds are changing their positions and those positions are getting refreshed and here in these systems additionally the position of every sensor is updated at each repetition.

Depending up on the fitness function the best control node is selected. If the fitness function is less than it is selected as control node. The nodes which are having less fitness function they consume less energy and the nodes which are having more fitness function they consume more energy. Residual energy is calculated for every node after every transmission. By calculating the residual energy we can have clarity of which node consumes less power and which node consumes more power. The node which is having more residual energy consumes less power and the node which is having less residual energy consumes more power.

The residual energy is the remaining energy that is left after the completion of the data transmission from the starting or initial point to the end user. The amount of energy that consumes till the completion of one transmission is called residual energy. We need to calculate the residual energy of each node after completion of the data transmission. By this also we can select the best control node for the cluster.

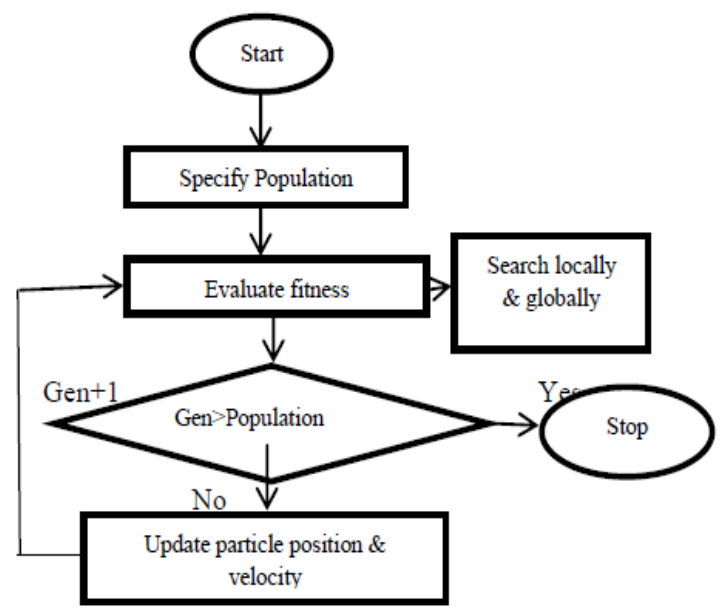

Fig. 3: Flow Diagram of PSO.

The above figure shows the flow chart how our pro- posed system is processed. First the particles are initialized each particle is having some energy and at first transmission of data packet some amount of energy is dissipated and now the update the position of the particle and calculating the amount of energy is consumed for the single transmission now for each particle there are two best values called pbest and gbest those values plays a major role in finding an optimal solution. Comparing the gbest with pbest and if the personal best value is better than the global best then the personal best value is equated to the global best like this the procedure continues until reaches the best solution.

For example a birds group is there and they need some food. The flock randomly searching for the food. They have no idea about the exact location of the food but, they have a little idea about the distance of the food location. In order to find out the exact location of the food by each bird is impossible or it is too difficult and takes more time. One bird among all knows the location that is the bird is nearer to the food. So, the other birds in the flock or swarm start following the neighbor birds until they reach the food. 
In PSO each single bird is considered as a problem solution or a particle in the swarm. Group of birds is considered as a swarm. All of the particles present in the swarm have its own fit- ness value evaluated from the fitness function. The particle in the swarm follows the other particles to find out the best solution for the optimization problem. Every particle in the swarm has a veloc- ity vector which shows the direction of the path and each particle has a position vector. The position and velocity should be updated at every iteration and each particle has its individual best that is called the personal best value pbest and all the particles combine and has a global best value called gbest these values are updated at every iteration until the particles reaches the destination. [12-15] [17-19].After this the position and velocity of the particles are updated at every iteration as follows

$\operatorname{vel}(\mathrm{i},:)=\mathrm{eta}^{*}\left(\mathrm{w}^{*} \operatorname{vel}(\mathrm{i},:)+\mathrm{c} 1 *\right.$ rand $($ size $($ gbest $)) .($ gbest-

pop(i,:))+c2*rand(size(gbest))*(pbest(i,:)-pop(i,:)))

$\operatorname{pop}(\mathrm{I},:)=\operatorname{pop}(\mathrm{I},:)+\operatorname{vel}(\mathrm{I},:)$

The equations (1) and (2) are used for the updating the particles position and velocity at the every single iteration.

Vel (I) is the particle velocity, Pbest and gbest are per- sonal best and global best respectively as stated before. Rand () is a random number in between o and $1 . \mathrm{C} 1 \mathrm{C} 2$ is learning factors and these are kept constant that is 2 .

First 2 joules of energy i.e.; power is given to the sensor nodes. Let us assume 50 particles are present. For each particle in the swarm we are giving 2 joules likewise 50times of 2 joules and starts transmitting the packet from the starting node to its neighbor. Now the initial power is getting dissipated slowly now we need to calculate the energy of the particle and need to update the position of the particle.

\subsection{Pseudo code}

For each particle

\{

Initialize particle

\}

Do until maximum iterations or minimum error criteria

\{

For each particle

\{

Calculate the data fitness value

If the fitness value is better than pbest

\{

Set pbest $=$ current fitness value

\}

If pbest is better than gbest

\{

Set gbest=pbest

\}

\}

For each particle

\{

Calculate particle velocity

Use gbest and velocity to update particle data

\}

Initially, we have to pick the particles in the swarm as a network or measurements. And after that we are giving some power to the particles called sensor nodes. The packet is transmit- ted from the source to the end client through the intra basic hubs in the system. At that point for transmission of the packet it re- quires some power. After transmission of the packet some amount of power is dissipated. In the first cycle this entire procedure is happened and same procedure is preceded until the point that it achieves the end client.

At each cycle we will update the position of the particle and find out the fitness function at every iteration. After calculate- ing the fitness function at every iteration we have to look at the fitness values of all the nodes and discovering the best fitness value. At this point we select the sensor node which is having the best fitness function as the leader of the group of the sensor nodes. The primary goal of the proposed approach is to discover the best control node for the group of nodes. By that we can minimize the power utilization of the hubs.

Control nodes consume more power than the common nodes in the network so by the proposed approach depending up on the fitness value we can select the best control node and by that we can reduce the power.

\section{Experimental results}

Here the results are embodied using Matlab as a plat- form. The X and $\mathrm{Y}$ axis are number of iterations and the fitness Value respectively. Depends up on the fitness value we can say that we reach the optimization solution.

Here proposed method contains different optimization func- tions and the graphs are given below.

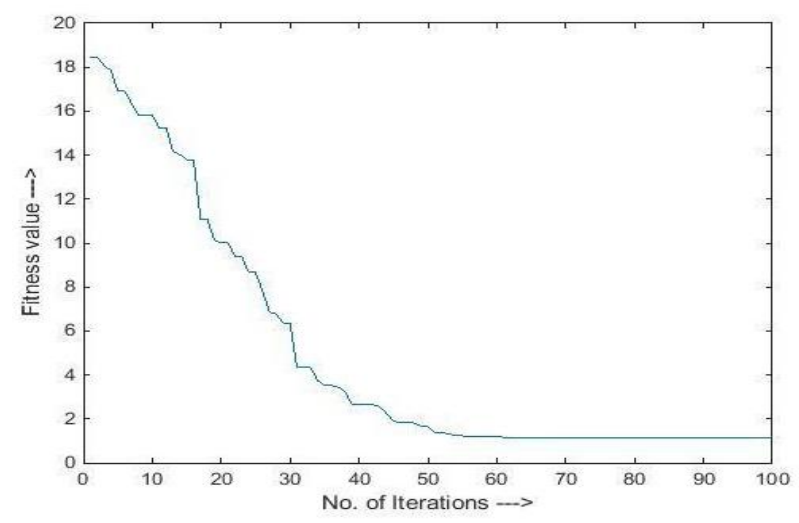

Fig. 4: Ackley Function.

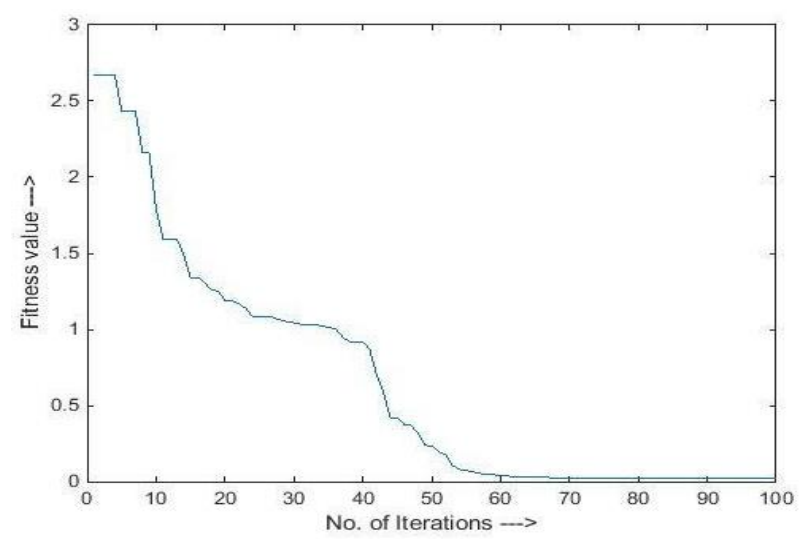

Fig. 5: Griwank Function.

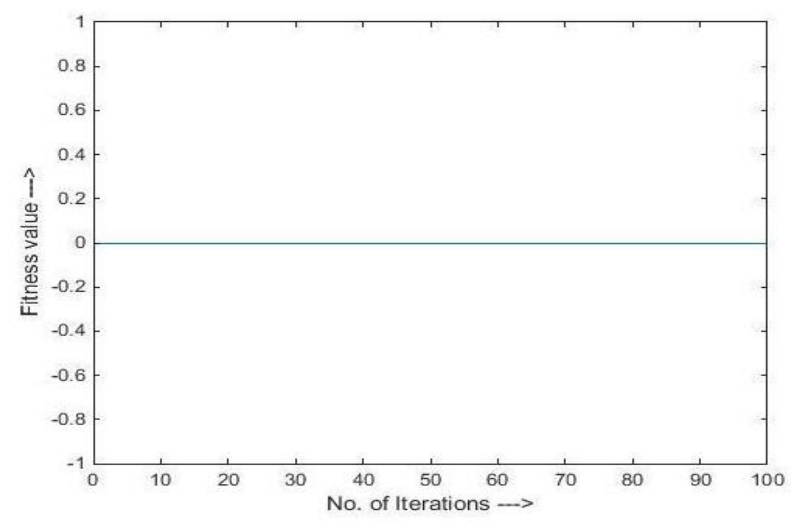

Fig. 6: Sphere Function. 


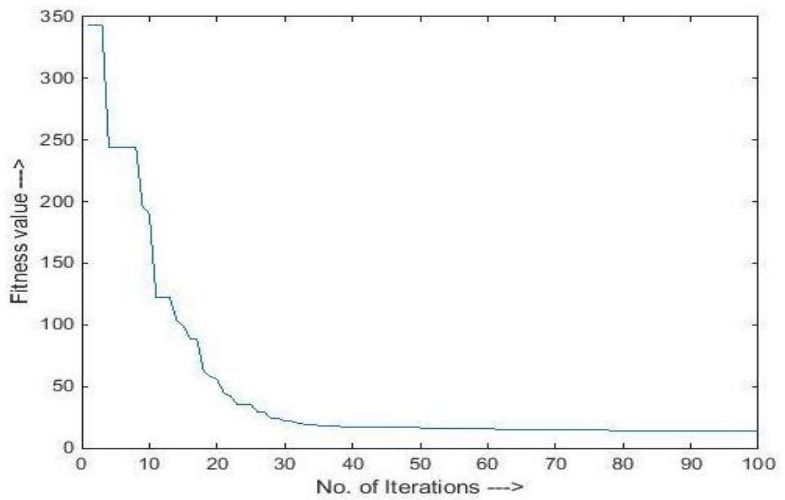

Fig. 7: Rosenbrock Function.

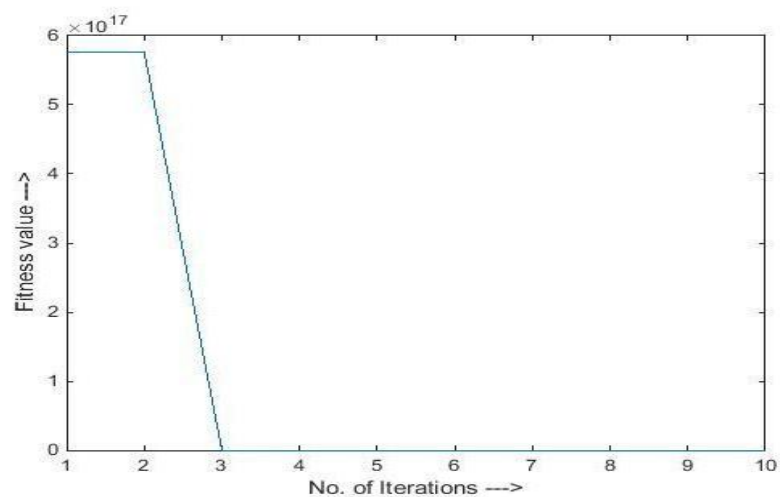

Fig. 8: Schwefel Function.

The above figures 4 to 8 shows the simulated results through particle swarm optimization using the different optimiza- tion functions. At every iteration the fitness value is getting re- duced and at some iteration we will get the minimum fitness value. The minimum the fitness value the highest the optimization solu- tion. By this we can get best optimization solution.

Table 1: Comparison of Different Optimization Functions

\begin{tabular}{llllll}
\hline Function & $\begin{array}{l}\text { Lower } \\
\text { Bound }\end{array}$ & $\begin{array}{l}\text { Upper } \\
\text { Bound }\end{array}$ & $\begin{array}{l}\text { Expected } \\
(\mathrm{min} .)\end{array}$ & $\begin{array}{c}\text { Obtained } \\
(\mathrm{min}) \text { value }\end{array}$ & Iterations \\
\hline Ackley & -35 & 35 & 1.222 & 1.1999 & 100 \\
Griwank & -100 & 100 & 0 & 0.0246 & 100 \\
Rosenbrock & -2 & 2 & 9.955 & 10.05 & 100 \\
Schwefel & -100 & 100 & 0 & 0 & 10 \\
Sphere & 0 & 10 & 0 & 0 & 100 \\
\hline
\end{tabular}

The above table shows the different Optimization func- tions comparison which is used for the power optimization. The expected result is approximately equals to the obtained results.

\section{Conclusion}

By the proposed approach called particle swarm optimization we can reduce the power consumption of the networks and the lifetime of the batteries can be increased. Since the batteries are nonrechargeable and by using the proposed approach we can choose the best head for the group of nodes called mote and by that we Can automatically reduce the energy consumption in WSN. Now days many researches are going on in the power consumption of networks by many researchers.

\section{References}

[1] Lisane brisolara, Paulo.r.ferreira, leandrosoares indrusiak, “Ap- plication modelling of performance evaluation of event- triggered wireless sensor networks", springer publications 2016.

[2] Jennifer Yick, Biswanth Mukherjee, Dipak Ghosal, "Wireless Sensor network survey", International Journal of Computer networks, Vol. 52, pp. 2292- 2330, 2008.

[3] Akylidiz, W. Su, Sankarasubramaniam, and E.Cayrici, "A sur- vey on sensor networks", IEEE Communications Magazine, Volume: 40 Issue: 8, August 2002, pp.102- 114.

[4] Dressler, F, Akan, O.B, "A survey on bio-inspired networking", Computer Network, Vol. 54, pp.881-900, 2010.

[5] Clerc, M. and Kennedy, J. "The particle swarm-explosion, sta- bility, and convergence in a multidimensional complex space", IEEE Transactions on Evolutionary Computation, Vol. 6, No. 1, pp. 5873. 2002.

[6] fariha nosheen, sadia bibi, salabath khan,"Ants colony based optimization scheduling algorithm", International conference on open source scheduling algorithm, 2013

[7] M. sasikala, A. Nithya, "Clustering in Wireless Sensor Net- works: A Survey, " International journal of computer trends and technology, vol 17 num 3, 2014.

[8] Mukul Pratap singh, Kunal gupta, "Techniques of Power Opti- mization for Wireless Sensor Networks", International Journal of Computer Applications, vol66, num3, 2013.

[9] Chandni, anjali bharti, "Optimization through Bio-inspired algorithms in Wireless sensor networks: survey and Future directions ", ISSN vol2, 2015

[10] Salma, Mohamed chedly, "Cluster based wireless sensor net- works optimization under energy constraints" ISSNIP, 2007.

[11] Shahrzad Dehghania , Mohammad Pourzaferanib , Behring Barekatainc , " comparison on energy efficient cluster based rout- ing algorithms in wireless sensor network" The third infor- mation systems international conference, 2015.

[12] C. Vimalarani, R. Subramanian, S. N. Sivanandam, "An En- hanced PSO Based Clustering Energy Optimization Algorithm for Wireless Sensor Network" scientific world journal, 2016.

[13] T. Luo, H.-P. Tan, and T. Q. Quek, "Sensor open flow: Ena- bling software-defined wireless sensor networks," IEEE Com- munications Letters, vol. 16, no. 11, pp. 1896-1899, Nov. 2012.

[14] Sandra sundra, lorena parra, Jaime Lloret,"systems and algo- rithms for wireless sensor networks based on Animal and natu- ral behavior".

[15] Debmalya Bhattacharya, R.Krishnamoorthy, "power optimiza- tion in wireless sensor networks", International journal of com- puter science, vol8, 2011.

[16] G.Hemavathy, S.Prabhu, "power optimization in wireless sen- sor networks using adjacent correction position algorithm", in- ternational journal of science and research, vol3, 2014.

[17] MM Chandane, S.G.Bhirud, S.V.Bonde "Energy Optimization in Wireless Sensor Network", springer, 2013.

[18] Hongliang Ren, Max Q H Meng, "Bio Inspired approaches for Wireless Sensor Networks", IEEE Conference on Mechatronics and Automation a Survey", pp. 762-768, 2014.

[19] Bonabeau, E., Dorigo, M. and Theraulaz, "Swarm intelligence", Oxford University Press, 1999. [19] R. Storn, K. Price, "Differ- ential evolution - a simple and efficient heuristic for global op- timization over continuous spaces", Journal of Global Optimi- zation, Vol. 11, pp. 341-359, 1997. 